\title{
Treatment Duration of Febrile Urinary Tract Infections
}

\author{
Willize E. van der Starre • Jaap T. van Dissel • \\ Cees van Nieuwkoop
}

Published online: 2 September 2011

(C) The Author(s) 2011. This article is published with open access at Springerlink.com

\begin{abstract}
Although febrile urinary tract infections (UTIs) are relatively common in adults, data on optimal treatment duration are limited. Randomized controlled trials specifically addressing the elderly and patients with comorbidities have not been performed. This review highlights current available evidence. Premenopausal, non-pregnant women without comorbidities can be treated with a 5-7 day regimen of fluoroquinolones in countries with low levels of fluoroquinolone resistance, or, if proven susceptible, with 14 days of trimethoprim-sulfamethoxazole. Oral $\beta$-lactams are less effective compared with fluoroquinolones and trimethoprim-sulfamethoxazole. In men with mild to moderate febrile UTI, a 2-week regimen of an oral fluoroquinolone is likely sufficient. Although data are limited, this possibly holds even in the elderly patients with comorbidities or bacteremia.
\end{abstract}

Keywords Febrile urinary tract infections - Acute pyelonephritis - Complicated urinary tract infection . Prostatitis · Antibiotics $\cdot$ Fluoroquinolones

\footnotetext{
W. E. van der Starre $(\bowtie) \cdot$ J. T. van Dissel · C. van Nieuwkoop Department of Infectious Diseases, Leiden University Medical Center, Postbox 9600, 2300 RC Leiden, the Netherlands e-mail: w.e.van_der_starre@lumc.nl

J. T. van Dissel

e-mail: j.t.van_dissel@lumc.nl

C. van Nieuwkoop

e-mail: c.vannieuwkoop@hagaziekenhuis.nl

C. van Nieuwkoop

Department of Internal Medicine, Haga Hospital,

Leyweg 275 ,

2545 CH The Hague, the Netherlands
}

\section{Introduction}

Urinary tract infections (UTIs) are among the most commonly encountered bacterial illnesses. In adults, UTIs can be classified into acute uncomplicated cystitis, acute uncomplicated pyelonephritis, complicated UTI, and acute complicated pyelonephritis. For men, several categories of prostatitis are distinguished [1-3]. The term "uncomplicated" usually reflects UTIs in premenopausal, non-pregnant women with no known anatomical or functional urological abnormalities or other comorbidities [4]. Some authors advocate to consider UTIs in postmenopausal women or women with well controlled diabetes mellitus without urological sequelae also to be "uncomplicated" [2]. Given these various classifications of UTIs in the literature, it likely makes more sense to classify these patients uniformly according to their presentation, in which fever reflects the presence of a parenchymal inflammation. From a scientific point of view, it is interesting to know whether the UTI involves the kidney, prostate, bladder, blood circulation, lymph nodes of the pelvis or a combination of all. But from a clinical point of view, febrile UTI should be considered as tissue inflammation of the urinary tract, whereas an exact anatomical distinction on clinical grounds can often not be made. In this article, we use febrile UTI as the clinical syndrome of interest because this is how patients present, and fever mainly determines the appropriate treatment. According to the above mentioned classifications, febrile UTI includes complicated UTI with fever, acute prostatitis, acute (un-)complicated pyelonephritis and, as has been suggested, the urosepsis syndrome [5].

The optimal treatment duration of febrile UTI has not been established yet, but current strategies recommend antimicrobial treatment for about 14 days in most patients $\left[4,6-9,10^{\bullet}\right]$. With the paucity of new antimicrobial 
classes in development, it is increasingly important to develop strategies to maintain or even increase the effectiveness of the available agents. Dose and regimen optimization represents one such strategy. Antimicrobials are associated with considerable side effects (eg Clostridium difficile infection with the use of fluoroquinolones) [11-13]. Moreover, longer duration of antimicrobial therapy with consequently selecting pressure on gut flora might lead to an enhanced risk of the selection of resistant strains [14]. In this respect, it is questionable whether the benefit of antimicrobial therapy for at least 14 days at the end of treatment still outweighs its potential side effects.

This review highlights the main research findings on treatment duration of community-acquired febrile UTI in non-pregnant adults. A summary of the pivotal studies among antimicrobial treatment duration of adults with febrile UTI is given in Table 1.

\section{Febrile UTI in Women}

The majority of UTIs in women are community-acquired uncomplicated cystitis that usually responds to a 3-day course of empiric antimicrobials. However, if UTIs are accompanied by fever, another treatment regimen will be required. Treatment guidelines for febrile UTI often only discuss acute uncomplicated pyelonephritis, defined as acute pyelonephritis in premenopausal, non-pregnant and otherwise healthy women without any comorbidity [4]. Although febrile UTI is a relatively common and potentially serious infection even in young women, few controlled trials have been conducted to define optimal therapy directly comparing the same drug given for different durations of therapy, though a number of publications compared various treatment durations between different antimicrobial agents. In one open label, single center randomized trial comparing 2 versus 6 weeks of oral therapy with trimethoprim-sulfamethoxazole or ampicillin in 60 women with acute uncomplicated pyelonephritis, 2-week therapy was as effective as 6-week therapy with either drug. However, shorter duration of treatment resulted in fewer adverse effects, less frequent selection of resistant strains, and lower costs [15]. Trimethoprimsulfamethoxazole was more effective than ampicillin due to more ampicillin-resistant strains and increased recurrence rate in ampicillin treated patients, even with susceptible strains. With ampicillin resistance in E. coli exceeding 50\% in many countries worldwide, ampicillin should not be used as empirical treatment of febrile UTI.

Nitrofurantoin should not be used for the treatment of febrile UTI because it does not achieve reliable tissue or serum levels [16].
Trimethoprim-Sulfamethoxazole and Fluoroquinolones

Talan and colleagues [17] showed that even therapy duration less than 14 days is effective in young, healthy women. This double-blind, multicenter randomized controlled trial compared a 7-day regimen of oral ciprofloxacin $500 \mathrm{mg}$ twice daily with a 14-day regimen of trimethoprimsulfamethoxazole $160 / 800 \mathrm{mg}$ twice daily for treatment of otherwise healthy women with mild to moderate pyelonephritis. An initial intravenous 1-g dose of ceftriaxone in the trimethoprim-sulfamethoxazole group or a 400-mg intravenous dose of ciprofloxacin in the ciprofloxacin group was allowed at the discretion of the doctor. At the 4-11 days post-therapy visit, ciprofloxacin had significantly higher microbiological (99\% vs $89 \%$, respectively) and clinical ( $96 \%$ vs $83 \%$, respectively) cure rates, regardless of whether an initial intravenous dose of ciprofloxacin was given. Among trimethoprim-sulfamethoxazole-treated women, microbiological eradication and clinical cure rates were significantly lower in women with a trimethoprimsulfamethoxazole-resistant strain compared with those with a susceptible one. However, an initial intravenous dose of ceftriaxone significantly improved microbiological cure rate and moderately improved clinical cure rate in women with a trimethoprim-sulfamethoxazole-resistant uropathogen. Bacteremia (all E. coli) was present in $5.5 \%$ of the patients; 2/10 trimethoprim-sulfamethoxazole-treated subjects had bacteriologic persistence, all four ciprofloxacin-treated subjects achieved bacteriologic cure.

Additional evidence for a 1 week regimen of fluoroquinolones as effective and safe treatment for healthy young women was provided by two other articles, describing the same study [18, 19]. This double blind, randomized multicenter trial included both men and women with complicated UTI (without fever) and acute pyelonephritis ( $20 \%$ to $30 \%$ ). Subgroup analysis of data about acute pyelonephritis [18] lend additional support that an oral 5day regimen of a once-daily fluoroquinolone (levofloxacin $750 \mathrm{mg}$ ) or a 10-day regimen of ciprofloxacin $500 \mathrm{mg}$ twice daily (in $6.1 \%$ after initial intravenous ciprofloxacin $400 \mathrm{mg}$ twice daily) might be effective for mild to moderate febrile UTI, even in those with bacteremia or complicating factors like obstruction or presence of a urinary catheter. Another pyelonephritis study in hospitalized men and women [20], prospectively comparing 10 days of norfloxacin $400 \mathrm{mg}$ twice daily with 10 days of ceftibuten $200 \mathrm{mg}$ twice daily, after receiving intravenous cefuroxime for 2 4 days in each group prior to randomization to the study drug, showed excellent clinical and bacteriological cure rates in both groups and lower bacterial relapse rates in patients treated with norfloxacin. The finding that a 1 week regimen of fluoroquinolones is both efficacious and safe for treatment of mild to moderate febrile UTI was further 


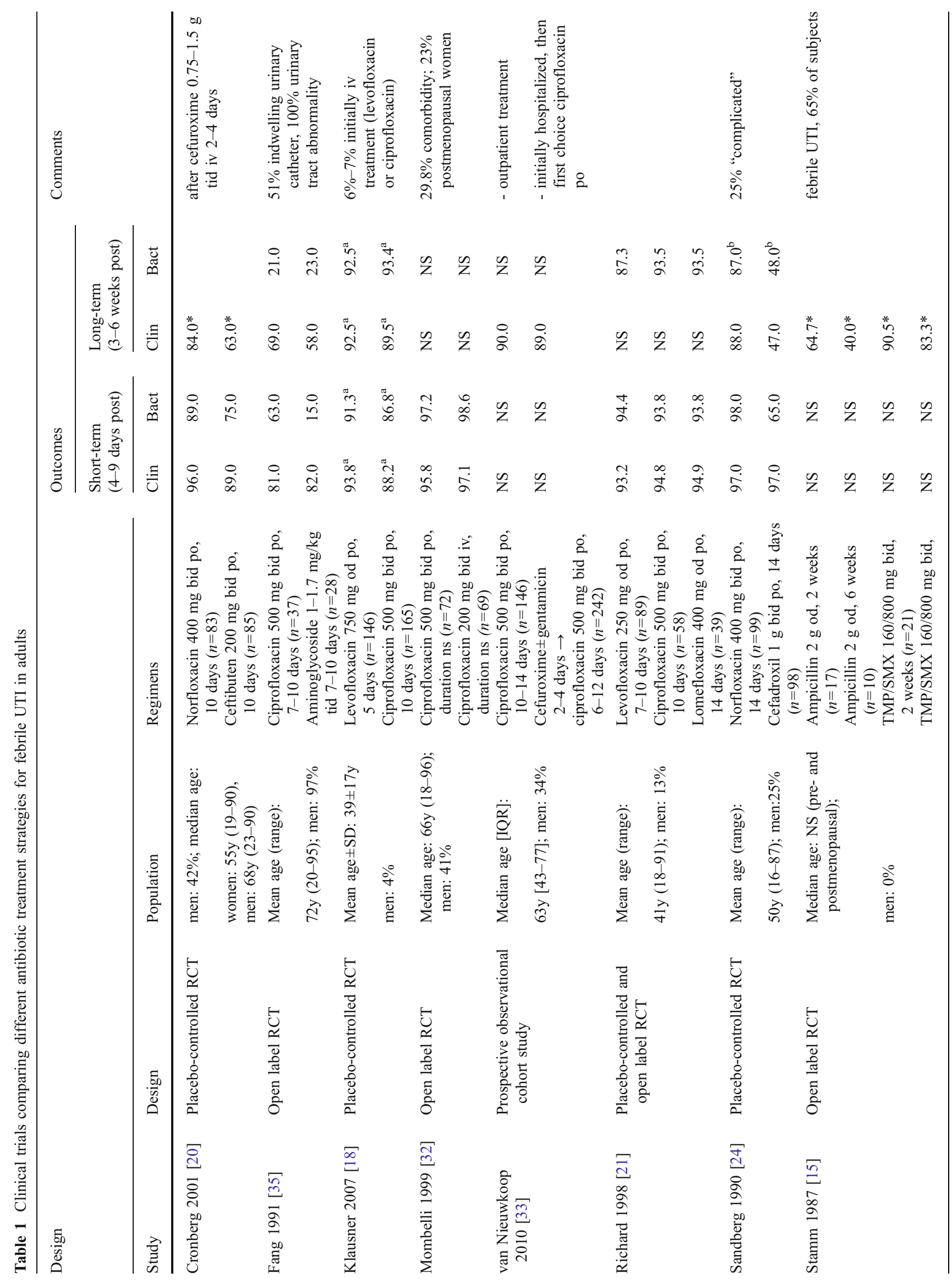


supported by a randomized comparative study demonstrating similar outcomes in comparing levofloxacin $250 \mathrm{mg}$ once daily, ciprofloxacin $500 \mathrm{mg}$ twice daily and lomefloxacin $400 \mathrm{mg}$ once daily, although in severe, invasive infections such a low dose of levofloxacin may result in marginal tissue and blood concentrations [21]. So, in regions with an acceptable low level of resistance to fluoroquinolones, this class of antimicrobials represents the preferred agents for empirical oral treatment of febrile UTI. With increasing rate of fluoroquinolone resistance in the last decade, the question raises at which threshold of resistance prevalence we should switch to an alternative agent for empirical treatment. Since clinical and bacterial outcomes with different levels of resistance are not well studied, the recommended threshold of $10 \%$ fluoroquinolone resistance prevalence is mainly based on expert opinion [4]. For some areas in the world, the prevalence of fluoroquinolone resistance is $>10 \%[22,23]$. Some experts advocate giving an initial dose of a long-acting parenteral antimicrobial, such as a 1-g dose of ceftriaxone or a consolidated 24-hour dose of an aminoglycoside (eg one 5-7 $\mathrm{mg} / \mathrm{kg}$ dose of gentamicin) in such situation [4].

\section{Oral $\beta$-Lactams in Women with Febrile UTI}

The data from Cronberg et al. [20] suggest inferior microbiological efficacy of oral cephalosporins compared with the fluoroquinolones, consistent with another Swedish study [24]. To our knowledge, there are no published studies on the efficacy of amoxicillin-clavulanate in the treatment of febrile UTI compared to trimethoprimsulfamethoxazole or a fluoroquinolone. However, a single-blind randomized trial in women with acute uncomplicated cystitis comparing a 3-day oral regimen of amoxicillin-clavulanate 500/125 mg twice daily with ciprofloxacin $250 \mathrm{mg}$ twice daily showed remarkable inferior clinical (60\% and $77 \%$, respectively) and microbiological ( $76 \%$ and $95 \%$, respectively) cure rates in the amoxicillin-clavulanate-treated group, even in women infected with susceptible strains $(60 \%$ in resistant versus $77 \%$ in susceptible strains). Most likely, the inferiority of $\beta$-lactams in the treatment of UTI is related to the lower rate of eradication of $E$. coli from the vagina in the amoxicillin-clavulanate group, maintaining a vaginal reservoir for infection [25]. Although not proven, these data are suggestive of an inferior efficacy of amoxicillinclavulanate in febrile UTI.

Optimal Treatment Duration in Young, Otherwise Healthy Women

To summarize the above data regarding treatment duration of febrile UTI in young, non-pregnant women without 
comorbidities, a 5-7 day oral regimen of a fluoroquinolone is both efficacious and safe in mild to moderate febrile UTI, given their superior clinical and bacteriological cure rates [17-19]. However, emerging fluoroquinolone resistance will possibly necessitate an initial dose of long-acting parenteral antimicrobial, such as a $1-\mathrm{g}$ dose of ceftriaxone or a consolidated 24-h dose of an aminoglycoside [4]. Trimethoprim-sulfamethoxazole is also highly efficacious in febrile UTI if caused by a trimethoprim-sulfamethoxaxole-susceptible uropathogen [15] but current high rates of resistance to trimethoprimsulfamethoxazole in many countries with corresponding failure rates for resistant strains make this agent an inferior choice for empirical therapy. When used empirically, combination with an initial intravenous dose of ceftriaxone resulted in improved bacteriological and clinical cure rates [17]. When the uropathogen is susceptible, current evidence support a 14-day regimen [17]; shorter treatment duration has never been well investigated. Oral $\beta$-lactams are likely less effective compared with fluoroquinolones and trimethoprimsulfamethoxazole $[15,20,24,25]$. When used, a total course of 10-14 days of therapy is recommended [4, 9, 10•, 26]. Probably, the same recommendations for treatment duration apply when the antimicrobial therapy was initially started intravenously (eg, in case of severe sepsis or inability to take oral medication).

\section{What Do the Guidelines Say?}

The above findings and treatment recommendations are in line with the recently updated IDSA-guideline [4]. The European Association of Urology (EAU) guideline recommends treating febrile UTI in premenopausal, otherwise healthy women with levofloxacin $750 \mathrm{mg}$ once daily for 5 days or ciprofloxacin $500 \mathrm{mg}$ twice daily for 7-10 days; a 14-day regimen is advised for all other oral antimicrobials and in all other febrile UTI patients [10•]. However, the guideline of the American College of Obstetricians and Gynaecologists (ACOG) recommends treating febrile UTI in all women (both pre and postmenopausal) for 14 days; fluoroquinolones are considered first line oral therapy and, in areas where resistance rates are "low," trimethoprimsulfamethoxazole is an acceptable alternative [9]. The guideline of the Dutch College of General Practitioners on the management of UTI recommends for outpatient treatment of adult patients with signs of tissue invasion, including fever, an oral regimen of either amoxicillinclavulanate $500 / 125 \mathrm{mg}$ three times a day, trimethoprimsulfamethoxazole $160 / 800 \mathrm{mg}$ twice daily, or a fluoroquinolone (eg norfloxacin $400 \mathrm{mg}$ twice daily or ciprofloxacin $500 \mathrm{mg}$ twice daily) for 10 days [26].

\section{Febrile UTI in "Complicated" Patients}

While treatment recommendations of febrile UTI are quite straightforward in young, healthy, non-pregnant women with short oral antimicrobial courses on an outpatient basis (provided they are able to take oral medication and have no severe sepsis), there is a lack of conformity on therapeutic approaches for other (sometimes referred as "complicated") categories of febrile UTI patients, like men, postmenopausal women, the elderly, or patients with urological abnormalities or indwelling urinary catheter. Oral antimicrobial treatment regimens and optimal treatment duration have hardly been studied in the latter categories. Most studies focus exclusively on febrile UTI in young, healthy women, or consist of a very heterogeneous study population with both women and men of all ages and a broad range of comorbidities, reducing the power and making subgroup analysis sometimes hardly to perform. There are no published trials specifically conceived to delineate optimal treatment duration in those categories, except for men. Currently, a randomized placebo-controlled study comparing a 7 to 14 day of antimicrobial therapy in consecutive patients with febrile UTI is ongoing to provide evidence within this category of "complicated"[27]. The results of this study are expected to become available in 2012 .

\section{Febrile UTI in Men}

UTI is very uncommon in otherwise healthy, young and middle-aged men. A Norwegian study [28] reported an estimated annual incidence of 6-8 UTIs per 10,000 men aged 21-50 years. Incidence increases with age because of urological abnormalities and instrumentation. Since more than $90 \%$ of men with febrile UTI have a concomitant infection of the prostate, as measured by transient increases in serum PSA and prostate volume,[29] the goal of treatment is not only to sterilize the urine but also to reach sufficient antimicrobial concentration in the prostate. Therefore, antimicrobials reaching free concentrations in prostatic fluid and prostatic tissue that exceed the minimum inhibitory concentrations of most common causative bacteria should be chosen for therapy. Fluoroquinolones have such favourable pharmacokinetic properties and antibacterial spectra [30]. Trimethoprim also achieves adequate concentrations in the prostate and is an alternative to fluoroquinolones provided the bacteria are susceptible to this antimicrobial [31]. As described earlier in this article and demonstrated in some mixed trials with a minority of men, therapy with $\beta$-lactams may result in lower cure rates in men with febrile UTI [20, 24].

There is an apparent lack of studies on optimal treatment duration of febrile UTI in men. We only found one study 
directly comparing different treatment durations in men [32]. In this open, prospective and randomized trial, 72 men with community-acquired febrile UTI (without a chronic indwelling catheter) were treated with ciprofloxacin $500 \mathrm{mg}$ twice daily for 2 or 4 weeks. All responded successfully with resolution of fever and symptoms. There was no significant difference in bacteriological cure rate 2 weeks post-treatment between patients treated for 2 or 4 weeks (89\% versus $97 \%, 95 \% \mathrm{CI}$ for difference in proportions $-3 \%$ to $19 \%$ ), nor after 1 year ( $59 \%$ versus $76 \%, 95 \% \mathrm{CI}-5 \%$ to $39 \%$ ). The cumulative clinical cure rate after 1 year was $72 \%$ and $82 \%$, respectively $(95 \% \mathrm{CI}-10 \%$ to $30 \%)$. Recurrences after 1 year comprised asymptomatic bacteriuria (48\%), symptomatic lower UTI (23\%), and another episode of febrile UTI (29\%). A tendency toward more recurrences in the 2-week group could be attributed to a larger proportion of men with urological lesions requiring surgical interventions (26\% versus 12\%) in that group. The results should be interpreted with some caution, given the wide confidence interval for the differences in cure rate, but this study suggests a 2-week course of ciprofloxacin $500 \mathrm{mg}$ twice daily may be an adequate treatment for febrile UTI in men.

Another Swedish study lent additional support for a 2-week regimen of oral fluoroquinolones in men [24]. In this randomized, double-blind trial, adult men and women with a presumptive diagnosis of acute pyelonephritis (defined as febrile UTI) were randomly assigned to receive a 14-day course of oral treatment with either norfloxacin $400 \mathrm{mg}$ twice daily or cefadroxil $1 \mathrm{~g}$ twice daily. Of 197 patients enrolled, $16(29.5 \%)$ men were treated with norfloxacin and $12(21.1 \%)$ with cefadroxil. In this subgroup, a 14-day regimen of norfloxacin was highly effective, regardless of the presence of bacteremia or complicating factors such as diabetes mellitus or urinary tract abnormalities, with significantly higher bacteriological cure rate than with cefadroxil, both at 310 days (100\% versus $73 \%$, respectively) and up to 2 months after cessation of treatment ( $88 \%$ versus $75 \%$, respectively).

The same results in men were obtained from a third Swedish trial, [20] which used step-down treatment; initial intravenous treatment with cefuroxime was followed by either norfloxacin $400 \mathrm{mg}$ twice daily or ceftibuten $200 \mathrm{mg}$ twice daily for 10 days. Thus, these studies provided evidence that men with mild to moderate febrile UTI may be safely treated at home with a 14 days regimen of an oral fluoroquinolone.

\section{Febrile UTI in the Elderly and Patients with Comorbidities}

Oral ciprofloxacin has a high bioavailability and a broad spectrum of activity against uropathogens. Therefore, Mombelli and colleagues [33] analyzed the efficacy of ciprofloxacin in the empirical management of severe febrile UTIs. In a multicenter prospective randomized study, patients with serious (including febrile) UTI were randomized in the hospital setting to empirical antibiotic treatment with ciprofloxacin received either orally or intravenously. Excluded were patients with severe sepsis, inability to take oral medication or renal obstructive disease. In this study, 141 patients participated; $39 \%$ were men, 42 had comorbidity (eg diabetes mellitus) and $35 \%$ had bacteremia. There were no infection-related deaths and no patients required an early change of antibiotics because of worsening clinical status during the initial empirical phase of treatment. The rates of microbiological failure $(3 \%$ in the oral versus $2 \%$ in the intravenous treatment group) and of unsatisfactory clinical response $(4 \%$ oral versus $3 \%$ intravenous) were low. A treatment change was eventually required in $14 \%$ of the patients assigned to the oral and $7 \%$ of the patients assigned to the intravenous regimen, mainly because of the isolation of enterococci or ciprofloxacinresistant organisms in pretherapy urine specimens. There were no differences in outcome between the two regimens in premenopausal versus postmenopausal women. Unfortunately, treatment duration in both groups was not stated. The authors concluded that in the hospital setting, oral ciprofloxacin is as effective as the intravenous regimen in the initial empirical treatment of serious UTI, including the bacteremic form. They hypothesize the oral regimen can be used for outpatient treatment even in serious UTI. Our own study among primary health care centers (PHCs) and emergency departments (EDs) supported this hypothesis [34-]. We performed a prospective observational cohort study including consecutive non-pregnant adults with febrile UTI. Of 395 evaluable patients, 153 were recruited by their GP and treated as outpatients; 146 (95\%) patients received oral ciprofloxacin $500 \mathrm{mg}$ twice daily for 10 14 days. The remaining 242 patients were recruited at EDs of which $35(14 \%)$ were treated as outpatients with oral ciprofloxacin and 207 (86\%) were admitted and empirically treated with cefuroxime \pm gentamicin. Median age was 63 years [IQR 42-77], 34\% were male and 58\% had comorbidity, all characteristics comparable between both groups. Bacteremia was present in $10 \%$ of the outpatients and $27 \%$ of the inpatients. During follow-up, $8(5 \%)$ of PHC group were hospitalized because of suspected deteriorating sepsis, progressive illness, or persistent symptoms; none of them required ICU-admission, nor were there any attributable deaths. Clinical cure rates at 30 days were high in both groups $(90 \%$ in $\mathrm{PHC}$ and $89 \%$ in ED-group, respectively) and persistent at least until 3 months follow-up. Bacteriological outcomes were not reported. Thus, the outcome of patients treated with oral ciprofloxacin on an outpatient basis suggests that among selected adults with febrile UTI, many can be 
safely treated at home using a 10-14 day regimen of oral fluoroquinolones, including men, the elderly, and patients with comorbidity or bacteremia.

Besides this observational study, there is remarkably little evidence in the literature regarding the optimal treatment duration of febrile UTI in the elderly or patients with comorbidity like diabetes mellitus or urological abnormalities, despite high prevalence, since most randomized trials have excluded patients with underlying systemic illnesses or urinary tract abnormalities. Current treatment durations are mainly based on expert opinion; recommendations often do not differ from that in non-diabetic, young and otherwise healthy patients $[4,9,10,35]$. Therefore, randomized therapeutic trials to define the optimal treatment duration in these patient categories are urgently needed.

\section{Cather-Related Febrile UTI}

A substantial part of the UTIs in elderly is catheter-related, but also in patients of any age with catheter-related febrile UTI it is not well known what the optimal duration of treatment is. We found one trial prospectively comparing treatment with either a parenteral aminoglycoside or oral ciprofloxacin $500 \mathrm{mg}$ twice daily for $7-10$ days in catheterized patients in a chronic care facility with complicated UTI (58.5\% febrile UTI) and urinary tract abnormalities (eg neurogenic bladder, obstruction), the majority being men [36]. Whether the urinary catheter has been replaced before start of treatment was not described. Clinical cure 5-9 days post-therapy was about $80 \%$ in both groups, and $69 \%$ versus $58 \% 28-30$ days post-therapy in the ciprofloxacin and aminoglycoside group, respectively. Bacteriological cure, defined as sterile urine culture, was achieved in the ciprofloxacin and aminoglycoside group in $63 \%$ and $15 \% 2-5$ days after first dose and in $21 \%$ and $23 \% 5-9$ days post-therapy, respectively. This trial, which is quite difficult to interpret because of uncertainty about catheter replacement before start treatment, suggests that ciprofloxacin 7-10 days at least clinically might be sufficient for treating catheter-related febrile UTI in men but more studies are needed to further explore this observation.

\section{Conclusions}

Based on the current evidence in the literature (Table 1), we conclude that premenopausal, non-pregnant and otherwise healthy women can be treated orally with 5-7 days of adequately dosed fluoroquinolones or, if proven susceptible to the urinary isolate, with 14 days of trimethoprimsulfamethoxazole. Oral $\beta$-lactams are probably less effective in comparison with fluoroquinolones and trimethoprim- sulfamethoxazole. In all other patients with febrile UTI, optimal treatment duration is still unknown and should, awaiting randomized controlled trials, probably be at least 14 days. Although approximately $10 \%$ of patients with uncomplicated febrile UTI have bacteremia, there is no evidence that bacteremia has prognostic significance or warrants longer therapy in an otherwise healthy individual when using an antibiotic regimen ensuring adequate tissue and blood concentrations.

Acknowledgement WEvdS gratefully acknowledges unrestricted funding by the gratitude foundation Franje 1.

Disclosure No potential conflicts of interest relevant to this article were reported.

Open Access This article is distributed under the terms of the Creative Commons Attribution Noncommercial License which permits any noncommercial use, distribution, and reproduction in any medium, provided the original author(s) and source are credited.

\section{References}

Papers of particular interest, published recently, have been highlighted as:

- Of importance

•- Of major importance

1. Stamm WE, Hooton TM. Management of urinary tract infections in adults. N Engl J Med. 1993;329:1328-34.

2. Naber KG. Experience with the new guidelines on evaluation of new anti-infective drugs for the treatment of urinary tract infections. Int J Antimicrob Agents. 1999;11:189-96.

3. Krieger JN, Nyberg Jr L, Nickel JC. NIH consensus definition and classification of prostatitis. JAMA. 1999;282:236-7.

4. •- Gupta K, Hooton TM, Naber KG, et al.: International clinical practice guidelines for the treatment of acute uncomplicated cystitis and pyelonephritis in women: A 2010 update by the Infectious Diseases Society of America and the European Society for Microbiology and Infectious Diseases. Clin Infect Dis 2011;52:e103-e120. This recently updated guideline gives an overview of treatment recommendations in premenopausal, otherwise healthy women with febrile UTI.

5. Kunin CM. Definition of acute pyelonephritis vs the urosepsis syndrome. Arch Intern Med. 2003;163:2393-4.

6. Rubenstein JN, Schaeffer AJ. Managing complicated urinary tract infections: the urologic view. Infect Dis Clin North Am. 2003; $17: 333-51$.

7. Ramakrishnan K, Scheid DC. Diagnosis and management of acute pyelonephritis in adults. Am Fam Physician. 2005;71:933-42.

8. Nicolle LE. Short-term therapy for urinary tract infection: success and failure. Int J Antimicrob Agents. 2008;31:40-5.

9. ACOG Practice Bulletin No. 91. Treatment of urinary tract infections in nonpregnant women. Obstet Gynecol. 2008;111:785-94.

10. - European Association of Urology: guidelines on urological infections. Available at www.uroweb.org. Accessed 15-4-2011. This guideline outlines concisely and to-the-point the current recommendations for treatment of febrile UTI. 
11. Patel NS. Fluoroquinolone use is the predominant risk factor for the development of a new strain of clostridium difficile-associated disease. BJU Int. 2007;99:1333-4.

12. Kazakova SV, Ware K, Baughman B, et al. A hospital outbreak of diarrhea due to an emerging epidemic strain of Clostridium difficile. Arch Intern Med. 2006;166:2518-24.

13. Loo VG, Poirier L, Miller MA, et al. A predominantly clonal multi-institutional outbreak of Clostridium difficile-associated diarrhea with high morbidity and mortality. $\mathrm{N}$ Engl J Med. 2005;353:2442-9.

14. Foxman B, Ki M, Brown P. Antibiotic resistance and pyelonephritis. Clin Infect Dis. 2007;45:281-3.

15. Stamm WE, McKevitt M, Counts GW. Acute renal infection in women: treatment with trimethoprim-sulfamethoxazole or ampicillin for two or six weeks. A randomized trial. Ann Intern Med. 1987;106:341-5.

16. Cunha BA. Nitrofurantoin: an update. Obstet Gynecol Surv. 1989;44:399-406.

17. Talan DA, Stamm WE, Hooton TM, et al. Comparison of ciprofloxacin (7 days) and trimethoprim-sulfamethoxazole (14 days) for acute uncomplicated pyelonephritis pyelonephritis in women: a randomized trial. JAMA. 2000;283:1583-90.

18. Klausner HA, Brown P, Peterson J, et al. A trial of levofloxacin $750 \mathrm{mg}$ once daily for 5 days versus ciprofloxacin $400 \mathrm{mg}$ and/or $500 \mathrm{mg}$ twice daily for 10 days in the treatment of acute pyelonephritis. Curr Med Res Opin. 2007;23:2637-45.

19. Peterson J, Kaul S, Khashab M, et al. A double-blind, randomized comparison of levofloxacin $750 \mathrm{mg}$ once-daily for five days with ciprofloxacin $400 / 500 \mathrm{mg}$ twice-daily for 10 days for the treatment of complicated urinary tract infections and acute pyelonephritis. Urology. 2008;71:17-22.

20. Cronberg S, Banke S, Bergman B, et al. Fewer bacterial relapses after oral treatment with norfloxacin than with ceftibuten in acute pyelonephritis initially treated with intravenous cefuroxime. Scand J Infect Dis. 2001;33:339-43.

21. Richard GA, Klimberg IN, Fowler CL, et al. Levofloxacin versus ciprofloxacin versus lomefloxacin in acute pyelonephritis. Urology. 1998;52:51-5.

22. Arslan H, Azap OK, Ergonul O, et al. Risk factors for ciprofloxacin resistance among Escherichia coli strains isolated from community-acquired urinary tract infections in Turkey. J Antimicrob Chemother. 2005;56:914-8.

23. Chaniotaki S, Giakouppi P, Tzouvelekis LS, et al. Quinolone resistance among Escherichia coli strains from communityacquired urinary tract infections in Greece. Clin Microbiol Infect. 2004;10:75-8.
24. Sandberg T, Englund G, Lincoln K, et al. Randomised doubleblind study of norfloxacin and cefadroxil in the treatment of acute pyelonephritis. Eur J Clin Microbiol Infect Dis. 1990;9:317-23.

25. Hooton TM, Scholes D, Gupta K, et al. Amoxicillin-clavulanate vs ciprofloxacin for the treatment of uncomplicated cystitis in women: a randomized trial. JAMA. 2005;293:949-55.

26. van Haaren KAM, Visser HS, van Vliet S, et al. NHG-Standaard Urineweginfecties: tweede herziening [Guideline of the Dutch College of General Practitioners on urinary tract infections: second revision]. Huisarts Wet. 2005;48:341-52.

27. van Nieuwkoop C, Van't Wout JW, Assendelft WJ, et al. Treatment duration of febrile urinary tract infection (FUTIRST trial): a randomized placebo-controlled multicenter trial comparing short (7 days) antibiotic treatment with conventional treatment (14 days). BMC Infect Dis. 2009;9:131.

28. Vorland LH, Carlson K, Aalen O. An epidemiological survey of urinary tract infections among outpatients in Northern Norway. Scand J Infect Dis. 1985;17:277-83.

29. Ulleryd P, Zackrisson B, Aus G, et al. Prostatic involvement in men with febrile urinary tract infection as measured by serum prostate-specific antigen and transrectal ultrasonography. BJU Int. 1999;84:470-4.

30. Wagenlehner FM, Naber KG. Fluoroquinolone antimicrobial agents in the treatment of prostatitis and recurrent urinary tract infections in men. Curr Infect Dis Rep. 2005;7:9-16.

31. Lipsky BA. Prostatitis and urinary tract infection in men: what's new; what's true? Am J Med. 1999;106:327-34.

32. Ulleryd P, Sandberg T. Ciprofloxacin for 2 or 4 weeks in the treatment of febrile urinary tract infection in men: a randomized trial with a 1 year follow-up. Scand J Infect Dis. 2003;35:34-9.

33. Mombelli G, Pezzoli R, Pinoja-Lutz G, et al. Oral vs intravenous ciprofloxacin in the initial empirical management of severe pyelonephritis or complicated urinary tract infections: a prospective randomized clinical trial. Arch Intern Med. 1999;159:53-8.

34. • van Nieuwkoop C, Van't Wout JW, Spelt IC, et al.: Prospective cohort study of acute pyelonephritis in adults: Safety of triage towards home based oral antimicrobial treatment. J Infect 2010;60:114-21. This observational study showed that a 2-week regimen of an oral fluoroquinolone might be sufficient, even in the elderly and patients with comorbidities.

35. Nicolle LE. Urinary tract infection in the elderly. J Antimicrob Chemother. 1994;33(Suppl A):99-109.

36. Fang GD, Brennen C, Wagener M, et al. Use of ciprofloxacin versus use of aminoglycosides for therapy of complicated urinary tract infection: prospective, randomized clinical and pharmacokinetic study. Antimicrob Agents Chemother. 1991;35:1849-55. 